\title{
Pancreas Transplantation: Past, Present, Future
}

\section{Shamik Dholakia, MRCS, MBBS, BSc, Shruti Mittal, MRCS, DPhil, MBBS, BSc, Isabel Quiroga, FRCS, DPhil, James Gilbert, FRCS, MA, Edward J. Sharples, FRCP, DPhil, Rutger J. Ploeg, FRCS, PhD, Peter J. Friend, FRCS, MD}

Nuffield Department of Surgical Sciences and Oxford Transplant Centre, University of Oxford and Oxford University Hospitals NHS Trust, $U K$.

\begin{abstract}
Diabetes is the pandemic disease of the modern era, with $10 \%$ of these patients having type 1 diabetes mellitus. Despite the prevalence, morbidities, and associated financial burden, treatment options have not changed since the introduction of injectable insulin. To date, over 40,000 pancreas transplants have been performed globally. It remains the only known method for restoring glycemic control and thus curing type 1 diabetes mellitus. The aim of this review is to bring pancreatic transplantation out of the specialist realm, informing practitioners about this important procedure, so that they feel better equipped to refer suitable patients for transplantation and manage, counsel, and support when encountering them within their own specialty. This study was a narrative review conducted in October 2015, with OVID interface searching EMBASE and MEDLINE databases, using Timeframe: Inception to October 2015. Articles were assessed for clinical relevance and most up-to-date content, with articles written in English as the only inclusion criterion. Other sources used included conference proceedings/presentations and unpublished data from our institution (Oxford Transplant Centre). Pancreatic transplantation is growing and has quickly become the gold standard of care for patients with type 1 diabetes mellitus and renal failure. Significant improvements in quality of life and life expectancy make pancreatic transplant a viable and economically feasible intervention. It remains the most effective method of establishing and maintaining euglycemia, halting and potentially reversing complications associated with diabetes.
\end{abstract}

(C) 2016 Elsevier Inc. All rights reserved. • The American Journal of Medicine (2016)

KEYWORDS: Diabetes; Euglycemia; Pancreas; Transplant

In 2012 the American Diabetes Association estimated \$176 billion in direct medical costs and a further $\$ 69$ billion due to reduced productivity. Diabetes is associated with multiple systemic complications and reduces survival significantly. ${ }^{1}$

The World Health Organization estimates 9\% of the global population, over $600,000,000$ people, to be diabetic, with a significant proportion of these patients requiring insulin and approximately $10 \%$ of this population having type 1 diabetes. $^{2}$ The annual mortality rate of patients with

Funding: No funding was needed for the study.

Conflict of Interest: We declare no conflicts of interest.

Authorship: SD initiated the concept; SD, SM, IQ, JG, and ES collected and analyzed results with SD writing the first draft of the manuscript; all authors contributed equally to the script, with RJP and PJF as the responsible supervisors.

Requests for reprints should be addressed to Shamik Dholakia, MRCS, MBBS, BSc, Nuffield Department of Surgical Sciences and Oxford Transplant Centre, University of Oxford and Oxford University Hospitals, NHS Trust, Oxford OX37LE, UK.

E-mail address: sham.dholakia@nhs.net insulin-induced hypoglycemic unawareness is estimated to be between $3 \%$ and $6 \%$, making it a significant problem. ${ }^{3}$

The aim of pancreas transplantation is to restore normoglycemia, curing diabetes and limiting the progression of complications associated with diabetes. In the majority of cases, pancreas transplantation is performed in individuals with type 1 diabetes that have end-stage renal disease, usually with uremia, retinopathy, progressive neuropathy, and hypoglycemic unawareness. In those diabetic patients without renal insufficiency, pancreas transplantation alone is performed to prevent life-threatening episodes of deep hypoglycemia, as well as to halt complications. ${ }^{4}$

Pancreas transplantation has not been classed as a lifesaving procedure, unlike other forms of transplantation, but may significantly improve quality of life, providing its recipients with increased independence in all aspects of life..$^{5}$ However, not being lifesaving implies that the morbidity and mortality associated with the procedure, including the complications of long-term immunosuppression, must be carefully weighed against any potential benefit. ${ }^{6}$ 
Since records began, over 40,000 pancreas transplants have been reported to the International Pancreas Transplant Registry, the majority of them in the US. It is estimated that over 7000 pancreatic transplants have been performed in Europe to date.

The number of patients registered on the active UK transplant list for a pancreas transplant alone (PTA), simultaneous pancreas-kidney (SPK), and islet transplant has increased significantly over the last 10 years, from 132 patients in 2005 to 270 patients in 2014. The number of pancreas donors and transplants has also increased steadily from 118 donors, resulting in 86 transplants in 2004-2005, to 456 donors and 246 transplants in 2013-2014.

\section{TYPES}

Currently there are 5 types of endocrine replacement using a donor pancreas and transplantation:

1. Pancreas transplant alone (PTA): Primarily for type 1 diabetics with frequent and severe episodes of hypoglycemia, who may be unaware, have impaired quality of life, or other issues that lead to noncompliance with insulin therapy. These patients tend to have adequate renal function and no uremia. Patients with glomerular filtration rate of $80-100 \mathrm{~mL} / \mathrm{min} / 1.73 \mathrm{~m}^{2}$ are unlikely to need a kidney transplant. ${ }^{8,9}$

2. Simultaneous pancreas-kidney transplant (SPK): Both organs come from the same deceased donor. SPK indications have been adapted by the UK Transplant Kidney and Pancreas Advisory Group and include type 1 diabetics with end-stage renal failure requiring dialysis or within 6 months. ${ }^{10}$

3. Pancreas-after-kidney transplant (PAK): Deceased donor pancreas transplantation is performed after a previous, and different, living or deceased donor kidney transplant. PAK transplant is indicated for those patients who would qualify for a PTA, those with a previously viable kidney allograft. The benefits include a reduced waiting time and reduced mortality rate when compared with SPK patients. ${ }^{11}$

4. Simultaneous deceased donor pancreas and live donor kidney transplant has the benefit of lower rate of delayed graft function than SPK and significantly reduced waiting times, resulting in improved outcomes compared with patients waiting for an SPK. ${ }^{12}$

5. Islet cell transplant: despite optimal insulin therapy, a proportion of patients are unable to control their hypoglycemia. It is this group who should be considered for islet cell transplantation. Even in the absence of insulin independence after transplantation, the presence of functioning islets appears to protect against refractory hypoglycemia. ${ }^{13}$

\section{HISTORY}

In 1893, an attempt was made to graft 3 pieces of sheep pancreas into the subcutaneous tissue of a diabetic child. Despite initial success, the patient died after 3 days due to severe ketoacidosis. ${ }^{14}$ The first human pancreas transplant was performed in December 1966 at the University of Minnesota by the team of Doctors Kelly, Lillehei, Merkel, Idezuki and Goetz, 3 years after the first kidney transplant. A pancreas, along with kidney and donor duodenum, was transplanted into a 28-year-old woman who became insulin independent, but died a short time later due to pulmonary embolism. ${ }^{15}$

Outcomes improved with time, and significantly when simultaneously performing an SPK transplant as the kidney graft functioned as a sentinel for rejection. In PTA, without a kidney, results were significantly inferior as rejection was often missed. Therefore, exocrine drainage managed by way of duodenocystostomy was initially favored so that urinary amylase could be measured as a way of monitoring the pancreas graft in patients, and the introduction of cyclosporin in 1983 significantly reduced rejection rates, improving patient survival. ${ }^{16,17}$

However, the loss of sodium bicarbonate passing out with urine created a metabolic acidosis, and in extreme cases, extracellular volume depletion required hospitalization. Other documented complications include chemical cystitis, urethritis, bladder leak, reflux pancreatitis, recurrent infections, bladder cancer, bladder stones, urethral strictures, urethral irritation, epididymitis, prostatitis, and prostatic abscess. ${ }^{18,19}$

As immunosuppression regimes improved, these complications since have been avoided through a switch back to enteric drainage where the head of the pancreas is joined to the small intestine using a duodenal conduit, which was demonstrated in the late $1990 \mathrm{~s}^{16}$ 


\section{PANCREAS DONATION}

The majority of pancreas grafts are retrieved from heartbeating deceased brain-dead donors, with an increase in number seen from non-heart-beating donors after circulatory death or from living donors. ${ }^{20}$ Overall, solid organs offered for pancreas transplantation remain underused due to the strict acceptance criteria of transplant units. The acceptance criteria include body mass index (BMI), age of donor, and alcohol and lifestyle factors, and vary between units.

Donor obesity is the most common reason for refusal, as donors with BMI $>35 \mathrm{~kg} / \mathrm{m}^{2}$ are more likely to have fatty infiltration of the pancreas, which is a risk factor for posttransplant complications and graft failure. ${ }^{21}$

Fatty infiltration of the pancreas is thought to affect the organ in 2 ways: the first is macrovesicular lipid droplets that form as the organ is cooled during retrieval, which contributes to microvasculature occlusion and graft thrombosis; the second is the direct inflammation associated with necrosis during ischemic reperfusion of this additional fat, leading to pancreatitis. ${ }^{22}$

Age is not an absolute contraindication to donation; however, although there is variation between units, donors over 60 years of age are less likely to be used for solid organ transplant and more likely to be used for islets, as are donors with BMIs over $30 \mathrm{~kg} / \mathrm{m}^{2}{ }^{16}$

\section{PATIENT ELIGIBILITY}

Patients are eligible for a pancreas transplant if they have, or are at high risk of developing, secondary complications of diabetes (eg, nephropathy, retinopathy, neuropathy) or lifethreatening, disabling hypoglycemia. Age is not an absolute contraindication to surgery; however, the probability that significant improvements are observed is reduced in older recipients, due to reduced time to reverse diabetic sequelae. It is important to be aware that the rejection rates are lower in younger recipients, and older recipients have an increased rate of postoperative complication and longer length of hospital stay. ${ }^{16}$ For these patients who are high risk for surgery, islet transplantation may be an option. ${ }^{23}$

\section{IMMUNOSUPPRESSION}

Immunosuppressive therapy initially began with nondepleting antibodies, but has since been replaced with antibody induction therapy as an optimum method of suppressing lymphocytes. ${ }^{24}$

Between 1980 and 2000, patients received triple immunosuppression with cyclosporine, azathioprine, and prednisone. However, after 2000, immunosuppression was intensified by induction therapy for both pancreas and kidney transplants with the use of anti T-cell induction using polyclonal anti-thymocyte globulin (ATG) or monoclonal anti-CD-25-directed therapy in the form of basiliximab or daclizumab antagonizing interleukin 2-receptors, and the Tcell-depleting antibody alemtuzumab known as Campath (Genzyme Corporation, Cambridge, Mass.). ${ }^{24,25}$
T-cell depletion during induction reduces the number and severity of biopsy-proven rejection episodes but does not have effect on survival rates. Over time, azathioprine has been replaced by mycophenolate mofetil (MMF), and cyclosporine by tacrolimus, both showing improved outcomes compared with their predecessors. ${ }^{26,27}$

Comparison of cyclosporine with tacrolimus in combination with MMF and steroid, plus induction with ATG in the EUROSPK 001 trial showed reduction in rates of severe rejection, with lower rates of pancreas graft loss at 3 years in the tacrolimus combination group. ${ }^{28}$ First-year results from the EUROSPK 002 study suggest that rates of acute rejection were similar in patients treated with tacrolimus plus sirolimus or tacrolimus plus MMF. ${ }^{29,30}$

Steroid-free regimes are geared at minimizing insulin resistance and wound infection. Retrospective comparison between alemtuzumab and ATG with tacrolimus and sirolimus maintenance suggests similar rates of pancreas graft rejection around $20 \% .{ }^{26}$ Campath is cheaper and associated with lower rates of viral infection than ATG and so is preferred by some transplant units. ${ }^{31}$

\section{REJECTION}

More than $90 \%$ of pancreas-rejection episodes are reversible in the absence of hyperglycemia. This can be identified using transplanted kidneys as surrogate markers if they are from the same donor, or direct biopsy of pancreas in response to changes in biochemical markers or clinical presentation. Hyperglycemia is thought to be associated with a low probability of reversal of rejection. ${ }^{16}$

Graft loss through rejection occurs as a result of alloimmunity or autoimmune recurrence, however, the latter remains difficult to determine. Rejection rates are around 5\%-25\% depending on what immunosuppressive regime is used. Acute rejection is an important risk factor for longterm graft loss as a result of chronic rejection (10\% for PTA and $4 \%$ for SPK). ${ }^{32}$ Intravenous immunoglobulin G and plasmapheresis have also been used to try and eliminate antibody during rejection episodes, with some sucess. ${ }^{33}$

\section{CURRENT SURGICAL PROCEDURE}

The traditional donor procedure involves en bloc dissection of the pancreas with the donor duodenum and spleen during retrieval, where the pancreas is then separated from the spleen. In the recipient, preparation of the pancreas involves a "Y" or trouser graft vascular anastomosis of the superior mesenteric artery and splenic artery using the donor iliac bifurcation graft and tying off small branches and lymphatics to gain hemostasis. ${ }^{34,35}$

The recipient undergoes a laparotomy. The iliac vessels are divided and prepared, while the graft portal vein anastomosis to the recipient's right common iliac vein or inferior vena cava gives systemic release of the endocrine pancreas (insulin, glucagon); the graft portal artery is anastomosed to the right common iliac artery, and the exocrine graft 
secretions are drained enterically using a side-to-side ileum-duodenum anastomosis ${ }^{35}$ (Figure).

Currently, in the USA, $>80 \%$ of all pancreas transplants use enteric drainage to manage exocrine pancreatic secretions, and more than $90 \%$ use systemic venous drainage. ${ }^{16,34}$

\section{CONTRAINDICATIONS AND COMPLICATIONS}

Absolute contraindications to surgery include significant cardiovascular disease with severe or noncorrectable coronary artery disease, cardiac ejection fraction of $<50 \%$, recent myocardial infarction, noncurable malignancyexcluding localized skin cancer, active sepsis, active peptic ulcer disease, severe psychiatric conditions leading to noncompliance, and inability to survive surgery or immunosuppression due to other significant comorbidity.

Relative contraindications include cerebrovascular event with long-standing impairment; human immunodeficiency virus; hepatitis $\mathrm{B}$ and $\mathrm{C}$ viruses; a BMI higher than 30; extensive vascular, aortic, and renal artery disease (making surgery high risk); excessive need for insulin $>1.5 \mathrm{U} / \mathrm{kg} / \mathrm{d}$; and continuous use of alcohol, smoking, and other drugs. ${ }^{16,28}$

Cardiac morbidity and postoperative infections are most common in pancreas recipients. Coronary artery disease should be treated as early as possible, with routine angiogram and echocardiograms becoming part of the preoperative assessment as $30 \%$ of asymptomatic patients with type 1 diabetes have substantial coronary stenosis on angiography. ${ }^{36}$

The most common cause of nonimmunogenic graft failure is thrombosis, with a reported incidence in literature of

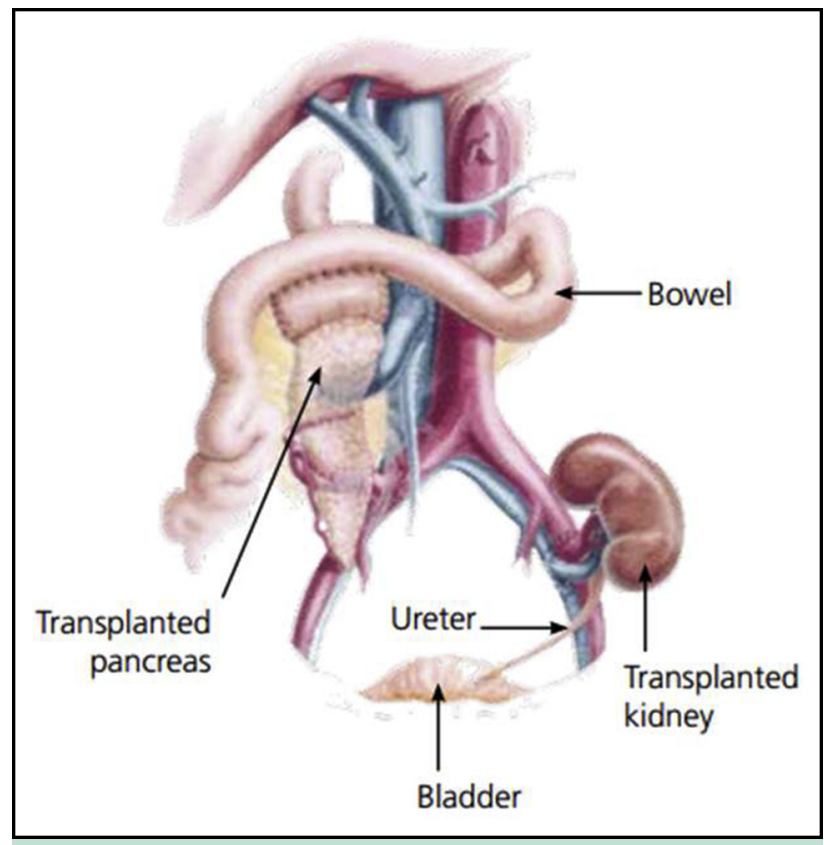

Figure Schematic diagram of simultaneous pancreas and kidney (SPK) transplant, with enteric drainage.
$10 \%$ to $35 \%$. Pancreatectomy is strongly associated with graft thrombosis, but the appropriate use of heparin, aspirin, and other anticoagulants have reduced the rate of thrombosis. $^{37}$

\section{OUTCOMES}

Improved outcomes and survival have been seen in patients and grafts, with some reversal of microvascular complications also seen. In SPK, patient survival has now reached more than $96 \%$ at 1 year after transplant, and more than $83 \%$ at 5 years after transplant. The best graft survival is seen in SPK transplant, with $86 \%$ pancreas and 93\% kidney graft function at 1 year. With PAK transplantation, pancreatic graft function reaches $80 \%$, and in PTA, pancreas graft function is $78 \%$ at 1 year. $^{28,38}$

International Pancreas Transplant Registry data analyses have consistently used insulin independence as the definitive criterion for graft survival. The estimated half-life (50\% function) of grafts has also improved, with SPK lasting 14 years, PAK 7 years, and PTA 7 years, with the longest surviving graft recorded for SPK with 26 years, 24 years for PAK, and 23 years for PTA. ${ }^{16,28,38}$

Preemptive SPK transplant recipients have better survival outcomes compared with those already established on dialysis. The main advantage of SPK transplant, apart from it being a cost-effective option, is the overall success rate of grafts, as acute rejection is easier to detect in both organs using serum creatinine as a marker. Acute rejection rates are similar for SPK and PAK, estimated at $4 \%$ and $4.3 \%$ respectively. ${ }^{38,39}$

\section{QUALITY OF LIFE AND SURVIVAL}

SPK transplantation has been shown in several studies to increase observed vs expected lifespan, when compared with kidney transplant alone; however, other studies have had conflicting results. SPK recipients had the highest longevity-23.4 years, compared with 20.9 years for live kidney transplant and 12.8 years for deceased donor kidney transplant. ${ }^{39,40}$

Evidence shows PAK recipients have improved patient and kidney graft survival as a result of pancreas transplantation, with significantly higher glomerular filtration rates seen in PAK patients compared with kidney transplant alone. ${ }^{16,37}$

Quality-of-life conclusions can be drawn only from patients with functioning or failed grafts and not from the 5\% that fail to survive beyond the first year. However, the evidence suggests there are large improvements in quality of life, a greater satisfaction with life, health, social and sex life, having more feelings of control and independence and better perceptions of social and mental health. ${ }^{41-43}$

\section{BIOLOGICAL AND METABOLIC IMPROVEMENT}

The diabetes control and complication trial showed that pancreas transplantation lowers glycated hemoglobin 
$\left(\mathrm{HbA}_{1 \mathrm{c}}\right)$ to within normal limits even after 10 years, with mean $\mathrm{HbA}_{1 \mathrm{c}}$ at 6 years being $6 \%$, compared with $7 \%$ of those being treated by intensified insulin regimes, thus restoring glycemic control and improving glucose counter regulation. Several studies have also reported improvement of lipid metabolism. ${ }^{44,45}$

Transplantation restores glucagon secretion, returns hepatic glucose production to normal, improves lipid profiles and insulin-mediated protein kinetics. Clinical improvements have been observed in diabetic nephropathy, neuropathy, gastroparesis, retinopathy (microvascular and macrovascular disease), cardiac function, and sexual function. $43,45,46$

There is also some evidence for patients with diabetic nephropathy, showing reversal of glomerular and cortical lesions due to diabetic nephropathy. One study showed almost complete normalization of glomerular structure 10 years post transplant. ${ }^{46,47}$

\section{FUTURE OF PANCREAS TRANSPLANTATION}

During the last decade, the number of pancreas transplants in the US has decreased by almost $20 \%$, whereas the number of pancreas transplants performed globally has increased. SPK has been associated with better pancreatic graft survival than PAK, which has translated in the decrease seen in the US mostly due to the decrease in primary PAK transplants, dropping by $50 \%$. The number of SPK transplants has decreased only slightly. ${ }^{48}$

The reasons for this decrease are not well understood, but are thought to be multifactorial. Analysis of the United Network for Organ Sharing/Scientific Registry of Transplant Recipients database suggests that fewer patients are being placed on the waiting list. This may be due to decreased rates of diabetic nephropathy development or delayed progression to end-stage renal failure. ${ }^{48,49}$

A further analysis on the decrease in recent years despite an increase in the number of patients awaiting transplantation shows that, although the overall number of abdominal organ donors has continued to increase, there has been a decrease in suitable pancreas donors as defined by age $\left(<60\right.$ years) and BMI $\left(<30 \mathrm{~kg} / \mathrm{m}^{2}\right)$, as many countries have an aging population and are fighting obesity epidemics. ${ }^{16,48}$

It is likely that adverse changes in the donor population are affecting allocation of suitable organs. Only approximately $15 \%$ of US deceased donors in 2013 donated a pancreas for transplantation, with some being allocated for islets or research. This is not a surprising trend, as the donor population becomes increasingly old, obese, and diabetic. $^{48,49}$

\section{ISLET CELL TRANSPLANTATION FOR TYPE 1 DIABETES}

Directed research into islet cell transplantation offers another potential solution in treating the diabetic population, and as it is a less invasive procedure, remains very appealing, especially for patients who are deemed high surgical risk. ${ }^{13,50}$

Currently, implantation remains a technically much simpler procedure, avoiding the complications and risks associated with solid organ transplant. However, the refinement and isolation of islet cells has been a limiting factor, with studies quoting from 300,000 to 750,000 islets required for $70 \%$ of patients to be insulin independent. ${ }^{51}$ Some studies suggest that around one million islets are needed for an average islet transplantation. Up to 6 purified donor pancreases are required to generate one million islets, which becomes an issue when donor organs are in short supply. ${ }^{51,52}$

Islet transplant results continue to improve since the introduction of the Edmonton immunosuppressive protocol, but long-term outcomes of islets have remained below those of pancreas transplantation. According to the collaborative islet transplant registry, insulin independence at 3 years post transplant improved from $27 \%$ in $1999-2002$ to $44 \%$ in 2007-2010. However, some of these results required multiple infusions to achieve results, and where compared with pancreas alone, transplantation at 5-year graft survival rates are no longer comparable. ${ }^{52,53}$

\section{FUTURE AND CHALLENGES}

As technology advances, new devices to improve blood glucose monitoring and insulin therapy are being developed to reduce the risk of hypoglycemia. Continuous glucose monitoring devices through real-time monitoring allow insulin pumps to continually secrete insulin through a closed loop system, and described as an artificial pancreas. Current limitations with delivery, absorption, and dosing still exist, ${ }^{54}$ but remain ever evolving. ${ }^{53}$

Advances over the last decade suggest that generating functional beta cells from stem cells is achievable. However, there are aspects of beta-cell development, including the signaling pathways, that instruct endocrine progenitor cells to differentiate into mature and functional beta cells, which remain poorly understood. Other issues include large-scale generation of transplantation-quality beta cells using costeffective models, as well as the risk of neoplasia. ${ }^{54,55}$

In this context, the concept of decellularization technology has been applied to produce whole organ-derived scaffolds by removing cellular content while retaining the extracellular matrix and necessary vascular and structural cues of the native organ, in hopes of rebuilding the whole organ. ${ }^{56}$ In the context of pancreas transplantation, this is still a significant distance away.

\section{CONCLUSION}

Pancreas transplantation has evolved from an experimental procedure to the gold standard of care for patients with type 1 diabetes and uremia. Currently, it remains the most effective method of establishing and maintaining 
euglycemia over the longer term, halting and potentially reversing many of the secondary complications associated with diabetes.

Significant improvements to quality of life and better life expectancy makes it, in the longer term, a lifesaving procedure compared with waiting candidates, makes pancreas transplantation a growing field of research with the aim to improve outcomes for patients, and possibly move toward offering nonuremic diabetics transplantation.

\section{References}

1. National Center for Chronic Disease Prevention and Health Promotion, Centers for Disease Control and Prevention (CDC). National Diabetes Statistics Report, 2014. Available at: http://www.cdc.gov/diabetes/pubs/ statsreport14/national-diabetes-report-web.pdf. Accessed December 11, 2015.

2. World Health Organization. Diabetes. Available at: http://www.who. int/mediacentre/factsheets/fs312/en/. Accessed December 18, 2015.

3. Nathan DM, Fogel H, Norman D, et al. Long-term metabolic and quality of life results with pancreatic/renal transplantation in insulindependent diabetes mellitus. Transplantation. 1991;52:85-91.

4. Gruessner RWG. Recipient procedures. In: Gruessner RWG, Sutherland DER, eds. Transplantation of the Pancreas. New York: Springer-Verlag; 2004:150-178.

5. NHS Blood and Transplant Media Services. Pancreas activity. Available at: https://nhsbtmediaservices.blob.core.windows.net/organdonation-assets/pdfs/pancreas_activity.pdf. Accessed December 2, 2015.

6. Gruessner RW, Sutherland DE, Gruessner AC. Mortality assessment for pancreas transplants. Am J Transplant. 2004:4:2018-2026.

7. Mel \& Enid Zuckerman College of Public Health, The University of Arizona. The International Pancreas Transplant Registry. Available at: https://publichealth.arizona.edu/research-project/ international-pancreas-transplant-registry. Accessed February 3, 2016.

8. Scalea JR, Butler CC, Munivenkatappa RB, et al. Pancreas transplant alone as an independent risk factor for the development of renal failure: a retrospective study. Transplantation. 2008;86:1789-1794.

9. Odorico JS, Voss B, Munoz DR. Kidney function after solitary pancreas transplantation. Transplant Proc. 2008;40:513-515.

10. Rayhill SC, D'Alessandro AM, Odorico JS, et al. Simultaneous pancreas-kidney transplantation and living related donor renal transplantation in patients with diabetes: is there a difference in survival? Ann Surg. 2000;231:417-423.

11. Gruessner AC, Sutherland DE, Dunn DL, et al. Pancreas after kidney transplants in posturemic patients with type I diabetes mellitus. $J$ Am Soc Nephrol. 2001;12:2490-2499.

12. Farney AC, Cho E, Schweitzer EJ, et al. Simultaneous cadaver pancreas living-donor kidney transplantation: a new approach for the type 1 diabetic uremic patient. Ann Surg. 2000;232:696-703.

13. Senior PA, Zeman M, Paty BW, et al. Changes in renal function after clinical islet transplantation: four-year observational study. Am J Transplant. 2007;7:91-98.

14. Lillehei RC, Simmons RL, Najarian JS, et al. Pancreatico-duodenal allotransplantation: experimental and clinical experience. Ann Surg. 1970;172:405-436.

15. Kelly WD, Lillehei RC, Merkel FK, et al. Allotransplantation of the pancreas and duodenum along with the kidney in diabetic nephropathy. Surgery. 1967:61:827-837.

16. Sutherland DE, Gruessner RW, Dunn DL, et al. Lessons learned from more than 1,000 pancreas transplants at a single institution. Ann Surg. 2001;233:463-501.

17. Mazur MJ, Rea DJ, Griffin MD, et al. Decline in native renal function early after bladder-drained pancreas transplantation alone. Transplantation. 2004;77:844-849.
18. Hickey DP, Bakthavatsalam R, Bannon CA, et al. Urological complications of pancreatic transplantation. J Urol. 1997;157:2042-2048.

19. Sollinger HW, Cook K, Kamps D, Glass NR, Belzer FO. Clinical and experimental experience with pancreaticocystostomy for exocrine pancreatic drainage in pancreas transplantation. Transplant Proc. 1984;16:749-751

20. Stegall MD, Dean PG, Sung R, et al. The rationale for the new deceased donor pancreas allocation schema. Transplantation. 2007;83: 1156-1161

21. Verma A, Papalois V. Evaluating steatosis in pancreatic transplant. Exp Clin Transplant. 2011;9:159-164.

22. Schulz T, Schenker P, Flecken M, et al. Donors with a maximum body weight of $50 \mathrm{~kg}$ for simultaneous pancreas-kidney transplantation. Transplant Proc. 2005;37:1268-1270.

23. Ridgway DM, White SA, Kimber RM, et al. Current practices of donor pancreas allocation in the UK: future implications for pancreas and islet transplantation. Transpl Int. 2005;18:828-834.

24. Kaufman DB, Iii GW, Bruce DS, et al. Prospective, randomized, multicenter trial of antibody induction therapy in simultaneous pancreaskidney transplantation. Am J Transplant. 2003;3(7):855-864.

25. Burke GW III, Kaufman DB, Millis JM, et al. Prospective, randomized trial of the effect of antibody induction in simultaneous pancreas and kidney transplantation: three-year results. Transplantation. 2004;77: 1269-1275.

26. Stegall MD, Simon M, Wachs ME, et al. Mycophenolate mofetil decreases rejection in simultaneous pancreas-kidney transplantation when combined with tacrolimus or cyclosporine. Transplantation. 1997;64: 1695-1700.

27. Bechstein WO, Malaise J, Saudek F, et al. Efficacy and safety of tacrolimus compared with cyclosporine microemulsion in primary simultaneous pancreas-kidney transplantation: 1-year results of a large multicenter trial. Transplantation. 2004;77:1221-1228.

28. Venstrom JM, McBride MA, Rother KI, et al. Survival after pancreas transplantation in patients with diabetes and preserved kidney function. JAMA. 2003;290:2817-2823.

29. Saudek F, Malaise J, Boucek P, et al. Efficacy and safety of tacrolimus compared with cyclosporin microemulsion in primary SPK transplantation: 3-year results of the Euro-SPK 001 trial. Nephrol Dial Transplant. 2005;20:3-10.

30. Arbogast H, Malaise J, Fernandez-Cruz L, et al; EURO SPK Group. Tacrolimus compared with cyclosporin microemulsion in primary simultaneous pancreas-kidney (SPK) transplantation: 3 year results of the EURO-SPK trial. Am J Transplant. 2008;5:267.

31. Gruessner RW, Kandaswamy R, Humar A, et al. Calcineurin inhibitorand steroid-free immunosuppression in pancreas-kidney and solitary pancreas transplantation. Transplantation. 2005;79:1184-1189.

32. Humar A, Khwaja K, Ramcharan T, et al. Chronic rejection: the next major challenge for pancreas transplant recipients. Transplantation. 2003;76:918-923

33. Cantarovich D, Vistoli F. Minimization protocols in pancreas transplantation. Transpl Int. 2009;22:61-68.

34. Sollinger HW, Odorico JS, Knechtle SJ, et al. Experience with 500 simultaneous pancreas-kidney transplants. Ann Surg. 1998;228: 284-296.

35. Iacovidou A, Hakim N. Recent advances in pancreatic transplantation. Exp Clin Transplant. 2013;11:471-474.

36. La Rocca E, Fiorina P, Di C. Cardiovascular outcomes after kidneypancreas and kidney-alone transplantation. Kidney Int. 2001;60:1964-1971.

37. Drachenberg CB, Papadimitriou JC, Farney A, et al. Pancreas transplantation: the histologic morphology of graft loss and clinical correlations. Transplantation. 2001;71:1784-1791.

38. Ojo AO, Meier-Kriesche HU, Hanson JA, et al. The impact of simultaneous pancreas-kidney transplantation on long-term patient survival. Transplantation. 2001;71:82-90.

39. Tyden G, Bolinder J, Solders G, et al. Improved survival in patients with insulin-dependent diabetes mellitus and end-stage diabetic nephropathy 10 years after combined pancreas and kidney transplantation. Transplantation. 1999;67:645-648. 
40. Bruce DS, Newell KA, Josephson MA, et al. Long-term outcome of kidney-pancreas transplant recipients with good graft function at one year. Transplantation. 1996;62:451-456.

41. Gross CR, Limwattananon C, Matthees BJ. Quality of life after pancreas transplantation: a review. Clin Transplant. 1998;12: 351-361.

42. Adang EM, Engel GL, Van Hooff JP, et al. Comparison before and after transplantation of pancreas-kidney and pancreas-kidney with loss of pancreas-a prospective controlled quality of life study. Transplantation. 1996;62:754-758.

43. Salonia A. Kidney-pancreas transplantation is associated with near-normal sexual function in uremic type 1 diabetic patients. Transplantation. 2011;92:802-808.

44. Katz H, Homan M, Velosa J, et al. Effects of pancreas transplantation on postprandial glucose metabolism. N Engl J Med. 1991;325: 1278-1283.

45. Robertson RP, Sutherland DE, Kendall DM, et al. Metabolic characterization of long-term successful pancreas transplants in type I diabetes. J Investig Med. 1996;44:549-555.

46. Biesenbach G, Konigsrainer A, Gross C, et al. Progression of macrovascular diseases is reduced in type 1 diabetic patients after more than 5 years successful combined pancreas-kidney transplantation in comparison to kidney transplantation alone. Transpl Int. 2005;18: 1054-1060.

47. Fioretto P, Mauer SM, Bilous RW, et al. Effects of pancreas transplantation on glomerular structure in insulin-dependent diabetic patients with their own kidneys. Lancet. 1993;342: 1193-1196.

48. Gruessner AC, Gruessner RW. Declining numbers of pancreas transplantations but significant improvements in outcome. Transplant Proc. 2014;46:1936-1937.

49. Redfield R, Sclea J, Odorico J. Simultaneous pancreas and kidney transplantation: current trends and future directions. Curr Opin Organ Transplant. 2015;20(1):94-102.

50. Ryan EA, Lakey JR, Rajotte RV, et al. Clinical outcomes and insulin secretion after islet transplantation with the Edmonton protocol. Diabetes. 2001;50:710-719.

51. Fiorina P, Shapiro AM, Ricordi C, et al. The clinical impact of islet transplantation. Am J Transplant. 2008;8:1990-1997.

52. Hering BJ. Achieving and maintaining insulin independence in human islet transplant recipients. Transplantation. 2005;79:1296-1297.

53. Shapiro AM, Ricordi C, Hering BJ. Edmonton's islet success has indeed been replicated elsewhere. Lancet. 2003;36:1242.

54. Rodbard D. Continuous glucose monitoring: a review of successes, challenges and opportunities. Diabetes Technol Ther. 2016;18(Suppl 2):S23-S213.

55. Mayhew CN, Wells JM. Converting human pluripotent stem cells into beta-cells: recent advances and future challenges. Curr Opin Organ Transplant. 2010;15(1):54-60.

56. Yagi H, Soto-Gutierrez A, Kitagawa Y. Whole-organ re-engineering: a regenerative medicine approach in digestive surgery for organ replacement. Surg Today. 2013;43(6):587-594. 